\title{
Simulation study using the spots deletion technique in spot scanning proton beam therapy for prostate cancers
}

\author{
NOBUYOSHI FUKUMITSU ${ }^{1}$, TOMOKATSU HAYAKAWA ${ }^{2}$, TOMOHIRO YAMASHITA ${ }^{3}$, \\ MASAYUKI MIMA $^{1}$, YUSUKE DEMIZU ${ }^{1}$, TAKESHI SUZUKI ${ }^{4}$ and TOSHINORI SOEJIMA ${ }^{1}$ \\ ${ }^{1}$ Department of Radiation Oncology, Kobe Proton Center, Kobe, Hyogo 650-0047; ${ }^{2}$ Division of Radiation Therapy, \\ Radiation and Proton Therapy Center, Shizuoka Cancer Center, Shizuoka, Shizuoka 411-8777; \\ ${ }^{3}$ Division of Medical Physics; ${ }^{4}$ Department of Anesthesiology, Kobe Proton Center, Kobe, Hyogo 650-0047, Japan
}

Received April 14, 2021; Accepted July 28, 2021

DOI: $10.3892 / \mathrm{mco} .2021 .2458$

\begin{abstract}
The aim of the present study was to investigate the effects on the dose distribution and beam delivery time in spot scanning proton beam therapy (PBT) incorporating the spot deletion technique. A spot scanning plan was created for 30 patients with prostate cancer. The plan was then modified via two processes: Spots with lower weighting depositions were deleted (process A) and spots that were distant from the clinical target volume (CTV) were deleted (process B). The dose distribution to the organs at risk (OAR), the expanded CTV (exCTV), which was defined by a uniform expansion of the CTV by a radius of $5 \mathrm{~mm}$, and the beam delivery time were compared among initial and modified plans. The $\mathrm{V}_{50 \text { Gy [relative biological effectiveness (RBE)] }}$ to the rectum and bladder, and $\mathrm{V}_{60 \mathrm{~Gy}(\mathrm{RBE})}$ to the urethral bulb, inhomogeneity index (INH) of the exCTV showed a difference $\left(\mathrm{P}=1.1 \times 10^{-14}, \mathrm{P}=6.4 \times 10^{-14}\right.$, $\mathrm{P}=2.7 \times 10^{-7}, \mathrm{P}=3.2 \times 10^{-17}$ ), although only changes by process $\mathrm{B}$ were significant. Modified plan by process $B$ showed the $\mathrm{V}_{50 \mathrm{~Gy}(\mathrm{RBE})}$ to the rectum and bladder decreased by $-2.4 \pm 1.6$ and $-2.3 \pm 1.4 \%$, and the $\mathrm{V}_{60 \mathrm{~Gy}(\mathrm{RBE})}$ to the urethral bulb decreased by $-15.9 \pm 19.4 \%$. The INH of the exCTV increased by $0.05 \pm 0.03 \%$. On the other hand, modification of the initial plan by process A did not affect the dose of the OAR, exCTV or beam delivery time. In spot scanning PBT, modification of the initial radiotherapy plan by systemic deletion of spots distant from the CTV could result in a dose reduction to the OAR.
\end{abstract}

\section{Introduction}

Proton beam therapy (PBT) is characterized by the emission of high radiation energy after penetration of the beam up to

Correspondence to: Dr Nobuyoshi Fukumitsu, Department of Radiation Oncology, Kobe Proton Center, 1-6-8 Minatoshima-Minamimachi, Kobe, Hyogo 650-0047, Japan

E-mail: fukumitsun@yahoo.co.jp

Key words: spot scanning, inhomogeneity index, beam delivery time, proton beam therapy, prostate cancer a certain depth $(1,2)$, and this therapeutic modality is widely used for the treatment of various cancers (3-5). Techniques for the delivery of PBT have advanced over the last few decades. One of the most representative advances is the development of the spot scanning technique using pencil beams. In the spot scanning irradiation technique, a lesion is visualized as a mass of points and each point is irradiated individually, unlike in conventional passive-scattered broad beam irradiation, in which a bundle of proton beams that are shaped to match the lesion is used. Scanning PBT is associated with superior beam flexibility that allows adaptation to complex-shaped targets. Other advantages are the reduced cost of manufacture of patient-specific apertures or compensators and the reduced time needed during delivery to change the devices (6-8). The number of facilities offering spot scanning PBT is growing rapidly worldwide. Spot scanning PBT has been applied for the treatment of prostate cancers $(9,10)$.

Several studies have investigated means to improve the image quality and/or shorten the beam delivery time, such as hardware or software modifications, including use of an improved collimator, spot resampling, or beam intensity adjustment $(6,11-18)$. Each of the methods has its own advantages, including reduction of the dose to the organs at risk (OAR), reduction of the out-of-field dose, improved optimization time, and shortened beam delivery time. However, some of these methods involve the use of special equipment that might entail huge costs and efforts for development and are not universally applicable in every facility. RayStation (RaySearch Medical Laboratories, Stockholm, Sweden) is a treatment planning system (TPS) which integrates a software package that allows definition of the doses to the target and OAR, management of the treatment plan and plan optimization, and provides delivery quality assurance (19). The TPS has the capability of allowing editing of the energy dose deposited on each spot even after optimization, such as adding, removing and/or multiplying the energy dose levels. Thus, it may be expected to allow modification of the dose distribution to make it closer to the ideal by deleting energy depositions of low importance.

The aim of this study was to investigate the effects of the spot deletion technique in spot scanning PBT in patients with prostate cancer, in whom the doses to the OAR are often controversial. 


\section{Materials and methods}

All the study procedures, which involved human participants, were conducted in accordance with the ethical standards of the institutional research committee and in compliance with the Declaration of Helsinki, and were approved by the Kobe Proton Center institutional review board. Patients' planning computed tomography data were used.

Simulation planning. Simulation planning was performed in 30 patients with prostate cancer (47-82 years old, T1 or $\mathrm{T} 2$ disease in all, according to the TNM classification). The clinical target volume (CTV) was defined as the whole prostate gland and the dose fraction to the isocenter of the CTV was 63 gray relative biological effectiveness [Gy (RBE)] with 21 fractions (20) The main parameter of the beam delivery system is shown in Table I; the beam direction was left and right opposition. The RayStation optimization algorithm is a sequential quadratic programming method that uses Broyden-Fletcher-Goldfarb-Shanno updates of the quasi-Newton approximation of the Hessian of the Lagrangian. Each beam included the range shifter of $0-6 \mathrm{~mm}$ water equivalent thickness made up of polyethylene. Robust optimization with a 3-mm setup and 3.5\% range uncertainty for CTV was used. The robustness parameters were decided by our accumulated set-up reproducibility data. Next, we calculated the perturbed doses due to the patient's positional variations $( \pm 3-\mathrm{mm}$ in 6 directions and $\pm 3.5 \%$ in range) and confirmed that the dose constraint was met. The dose constraints were determined by referring to a multi-institutional research on Japanese proton beam facilities and a treatment plan was created (initial plan). Default optimization parameters and clinical goal are shown in Table II. The maximum number of iteration was 40 .

Then, the initial plan was modified by 2 processes: process $\mathrm{A}$, in which spots with lower weighting depositions were deleted, and process $\mathrm{B}$, in which spots that were distant from the CTV were deleted. In process A, relative energy spots were deleted in ascending order by $0.01 \%$. In process $\mathrm{B}$, spots located away from the CTV were deleted at 1-mm intervals (Fig. 1). We had checked, in a preliminary study conducted prior to this study, that the deletion of spots more than $15 \mathrm{~mm}$ away from the CTV had little effect on the plan quality. Thus, we started inward from a distance of $15 \mathrm{~mm}$ in process $\mathrm{B}$. The step sizes in each process was such that they were clear-cut and the classification of the two processes would be consistent. In both processes, the spot deletion procedure was performed for each beam individually and the optimization was repeated after that. After spot deletion procedure, we calculated re-optimized plan and perturbed dose as same as the initial plan and confirmed that the dose constraint was met. Although this is a simulation study, we routinely setup using bone structure at first and fine-tune using implanted a pair of metallic markers by anterior and lateral X-ray fluoroscopic images in clinical practice. The metallic markers $(0.28 \mathrm{~mm}$ diameter, $20 \mathrm{~mm}$ length) are implanted in bilateral lobes where one is ventral and the other is dorsal side of the prostate gland.

Data analysis. Both processes were continued while every dose constraint was maintained. We investigated the dose distribution to the OAR, to the target, and the beam delivery time. In the analysis of the dose distribution to the OAR, we calculated the $\mathrm{V}_{50 \mathrm{~Gy}(\mathrm{RBE})}$ and $\mathrm{D}_{\max }$ of the rectum, $\mathrm{V}_{50 \mathrm{~Gy}(\mathrm{RBE})}$ and $\mathrm{D}_{\max }$ of the bladder, and $\mathrm{V}_{60 \mathrm{~Gy}(\mathrm{RBE})}$ and $\mathrm{D}_{\max }$ of the urethral bulb. In the analysis of the dose distribution to the target, the inhomogeneity index (INH) were calculated, as follows (21):

$$
I N H=\frac{D_{5}-D_{95}}{D_{\text {pre }}}
$$

The expanded CTV (exCTV) was defined by a uniform expansion of the CTV by a radius of $5 \mathrm{~mm}$ for only plan comparison referring the method of Kirk et al (22). $\mathrm{D}_{5}$ and $\mathrm{D}_{95}$ are the doses to 5 and $95 \%$ of the exCTV, and $\mathrm{D}_{\text {pre }}$ is the prescription dose. The beam delivery time was calculated as the sum of the time spent on each layer and the time interval between layers. The time spent in each layer was calculated as follows:

$$
\text { Time spent on layer }=\frac{\text { Monitor Units }}{\text { Intensity } \mathrm{x} \text { duty cycle }}
$$

Intensity means Monitor Unit/time and duty cycle means ratio of beam-on time/beam-on + off time.

We added examination to the patients with spacer implantation because separation effect due to the spacer might make it unnecessary to delete spots like process B. We examined whether the process $\mathrm{B}$ could reduce the dose of the rectum in 8 patients with SpaceOAR ${ }^{\circledR}$ System (Augmenix, Inc.) implantation as adding trial.

Statistics. The values represent the means \pm standard deviation. Single-factor ANOVA with Bonferroni's correction was used for comparing the data between the initial and modified plan, and minimum value in all modified plans was used as the value of modified plan in the OAR dose and beam delivery time comparison and maximum value was used in INH comparison. $\mathrm{P}<0.05$ was considered to indicate a statistically significant difference.

\section{Results}

In the plan modification by process $\mathrm{A}$, that is, deletion of lower weighting spots, relative energy doses with weights of $0.02-0.1 \%$ were deleted $(0.02 \%$ : $5 ; 0.03 \%$ : $8 ; 0.04 \%$ : 10 ; $0.05 \%: 4 ; 0.06 \%: 2 ; 0.1 \%$ : 1 patients). In the plan modification by process $\mathrm{B}$, that is, deletion of distant spots, energy spots were deleted from 13-9 mm away from the CTV (13 mm: 5; $11 \mathrm{~mm}$ : 19; $10 \mathrm{~mm}$ : 1; $9 \mathrm{~mm}$ : 5 patients). Table III shows a summary of the data. Figs. 2-4 show the changes in the data obtained by modification of the initial treatment plan by processes $\mathrm{A}$ and $\mathrm{B}$.

Dose distribution to the OAR. The $\mathrm{V}_{50 \mathrm{~Gy}(\mathrm{RBE})}$ of the rectum was between 3.5 and $16(11.1 \pm 3.1) \%$ as per the initial plan. It finally changed to between 3.1 and $15.8(11.3 \pm 3.2) \%$ following the initial plan was modified by process $\mathrm{A}$; following modification of the initial plan by process $\mathrm{B}$, it finally changed to between 3.4 and $14.9(8.9 \pm 2.7) \%$. The $\mathrm{V}_{50 \mathrm{~Gy} \text { (RBE) }}$ of the bladder was between 4.4 and $17.5(9.7 \pm 3.6) \%$ as per the initial plan. It finally changed to between 4.4 and $17.1(10.1 \pm 3.7) \%$ following the initial plan was modified by process $\mathrm{A}$; following modification of the initial plan by process $\mathrm{B}$, it finally changed to between 3.5 and $16(7.4 \pm 3) \%$. The $\mathrm{V}_{60 \mathrm{~Gy}(\mathrm{RBE})}$ of the urethral 
Table I. Beam parameters.

\begin{tabular}{lc}
\hline Parameters & Value \\
\hline Energy, MeV & $70.7-235$ \\
Energy steps, steps & 92 \\
Pulse frequency, Hz & $1 / 2.8$ \\
Field size, cm & $20 \times 15$ \\
Source-axis distance, X, Y, m & $2.696,3.029$ \\
Scanning speed, X, Y, mm/msec & 60,120 \\
Spot size at isocenter in air, mm, one $\sigma$ & $3.3-12$ \\
Dose rate, Gy/(l min) & 1 \\
\hline
\end{tabular}

bulb was between 80.6 and $100(99.2 \pm 3.5) \%$ as per the initial plan. It finally changed to between 81.4 and $100(99.2 \pm 3.4) \%$ following the initial plan was modified by process $\mathrm{A}$; following modification of the initial plan by process $\mathrm{B}$, it finally changed to between 33.6 and $100(83.4 \pm 19.7) \%$. Thus, the $\mathrm{V}_{50 \text { Gy (RBE) }}$ of the rectum, $\mathrm{V}_{50 \mathrm{~Gy}(\mathrm{RBE})}$ of the bladder, and $\mathrm{V}_{60 \mathrm{~Gy}(\mathrm{RBE})}$ of the urethral bulb showed a significant difference among groups $\left(\mathrm{P}=1.1 \times 10^{-14}, 6.4 \times 10^{-14}\right.$, and $2.7 \times 10^{-7}$, respectively) and following modification of the initial treatment plan by process $\mathrm{B}$ showed significant decrease, while no significant difference was noted with modification by process $\mathrm{A}$.

$\mathrm{D}_{\max }$ of the rectum was between 54.2 and $64(63 \pm 1.8) \mathrm{Gy}$ (RBE) as per the initial plan. It finally changed between 53.7 and $65.2(63.2 \pm 2) \mathrm{Gy}$ (RBE) following the initial plan was modified by process $\mathrm{A}$. While, following modification of the initial plan by process B, it finally changed between 54.2 and $64.2(62.7 \pm 1.9) \mathrm{Gy}(\mathrm{RBE}) . \mathrm{D}_{\max }$ of the bladder was between 62.6 and $64.4(63.6 \pm 0.4) \mathrm{Gy}(\mathrm{RBE})$ as per the initial plan. It finally changed to between 62.4 and $65.9(63.8 \pm 0.6) \mathrm{Gy}$ (RBE) following the initial plan was modified by process $\mathrm{A}$. While, following modification of the initial plan by process $B$, it finally changed to between 61.2 and $64.8(63.5 \pm 0.9)$ Gy (RBE). $\mathrm{D}_{\text {max }}$ of the urethral bulb was between 63.8 and 65.5 $(64.4 \pm 0.4)$ Gy $(\mathrm{RBE})$ as per the initial plan. It finally changed to between 63.6 and $64.7(64.2 \pm 0.3) \mathrm{Gy}$ (RBE) following the initial plan was modified by process A. While, following modification of the initial plan by process $\mathrm{B}$, it finally changed to between 62.7 and $65.0(63.9 \pm 0.5)$ Gy $(\mathrm{RBE})$. Thus, the $\mathrm{D}_{\max }$ of the rectum and bladder showed a significant difference among groups $\left(\mathrm{P}=6.1 \times 10^{-4}\right.$ and $4.9 \times 10^{-3}$, respectively), but no significant difference was noted between initial plan and modification by process A or B (Fig. 2).

Dose distribution to the target. The INH was between 0.03 and $0.1(0.04 \pm 0.01)$ as per the initial plan. It finally changed to between 0.03 and $0.1(0.05 \pm 0.01)$ following modification of the initial plan by process $\mathrm{A}$. While, following modification of the initial plan by process $\mathrm{B}$, it finally changed to between 0.04 and $0.14(0.08 \pm 0.03)$. The INH showed a significant difference among groups $\left(\mathrm{P}=3.2 \times 10^{-17}\right)$ and following modification of the initial treatment plan by process $\mathrm{B}$ showed significant increase (Fig. 3).

Beam delivery time. The beam delivery time was between 238.4 and $424.4(302.6 \pm 47.2)$ sec as per the initial plan. It finally changed to between 232.8 and $415.7(296.9 \pm 46.5) \mathrm{sec}$ following modification of the treatment plan by process $\mathrm{A}$. While, following modification of the plan by process $\mathrm{B}$, it

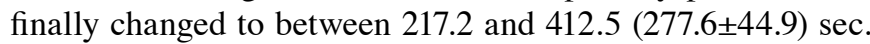
The beam delivery time showed a significant difference among groups $\left(\mathrm{P}=2.5 \times 10^{-18}\right)$, but no significant difference was noted with between initial plan and modification by process $\mathrm{A}$ or B (Fig. 4).

Adding analysis to the patients with spacer implantation. The $\mathrm{V}_{50 \mathrm{~Gy}(\mathrm{RBE})}$ of the rectum was $1.7 \%$ as per the initial plan. It changed to $1.1 \%$ in $15 \mathrm{~mm}, 1.1 \%$ in $13 \mathrm{~mm}, 0.8 \%$ in $11 \mathrm{~mm}$, and $0.4 \%$ in $9 \mathrm{~mm}$ plans. $\mathrm{D}_{\max }$ of the rectum was also reduced from $49.7 \mathrm{~Gy}(\mathrm{RBE})$ in initila plan to $48.8 \mathrm{~Gy}(\mathrm{RBE})$ in $15 \mathrm{~mm}$, $46.6 \mathrm{~Gy}(\mathrm{RBE})$ in $13 \mathrm{~mm}, 41.0 \mathrm{~Gy}(\mathrm{RBE})$ in $11 \mathrm{~mm}$ and $37.2 \mathrm{~Gy}(\mathrm{RBE})$ in $9 \mathrm{~mm}$ plans. On the other hand, $\mathrm{D}_{95}$ of the CTV was $100 \%$ in initial plan and $100 \%$ in $15-11 \mathrm{~mm}$ plans and $99.9 \%$ in $9 \mathrm{~mm}$ plan.

Number of layers, spots and spots distribution. The number of layers was $34 \pm 3.1,32.7 \pm 3.2$, and $32 \pm 3.3$ and the number of spots was $2288 \pm 632,1461 \pm 472$, and $1575 \pm 555$ in initial plan, process A and process B (Fig. 5). Fig. 6 shows one example of spots distribution. Relatively high-weighted deposition spots for each beam can be seen in the area beyond the CTV and relatively low-weighted deposition spots are found in and around the CTV.

Case presentation. An 80-year-old man with prostate cancer. Deletion of spots was continued to the level of $9 \mathrm{~mm}$ from the CTV via process B. As the spot deletion range moved inward, the radiation doses to the rectum and bladder decreased. Also, the elevated and reduced dose distribution areas became mottled in the CTV and exCTV, which caused dose inhomogeneities (Figs. 7 and 8).

\section{Discussion}

It is extremely important to reduce late adverse events in the treatment of cancers with a long survival prognosis, such as prostate cancer. Hou et al conducted a meta-analysis of 6 large randomized trials that included a total of 2822 patients, and reported that Grade 2 or more severe late GI toxicity occurred at a frequency of $18.6 \%$ in the cases receiving conventional radiotherapy and at a frequency of $28 \%$ in the cases receiving high-dose radiotherapy (23). They also reported that Grade 2 or more severe late GU toxicity occurred at a frequency of $19.5 \%$ in cases receiving conventional radiotherapy and at a frequency of $22.6 \%$ in cases receiving high-dose radiotherapy. In a more recent trial, Jolnerovski et al reported that the frequencies of Grade 2 or more severe late GI and GU toxicity at 5 years were 6.3 and $25.3 \%$ (24). They found from subgroup analyses that the total radiation dose was associated with the rate of GI toxicity and that the rate of GU toxicity was associated with the $\mathrm{D}_{\max }$ and $\mathrm{D}_{2 \%}$ (24). A close relationship exists between the dose and late toxicities, and rectal toxicity is particularly commonly associated with a higher dose volume (25-27). We examined the $\mathrm{V}_{50 \text { Gy (RBE) }}$ of the rectum and bladder and $\mathrm{V}_{60 \mathrm{~Gy}(\mathrm{RBE})}$ of the urethral bulb as an indicator of high-dose volume besides $D_{\max }$ in this study. Since the CTV had robustness, it was difficult to 
Table II. Optimization parameters and dose constraints.

\begin{tabular}{|c|c|c|c|}
\hline Organs & Optimization parameters & Weight & Clinical goal \\
\hline CTV & Uniform dose, $63 \mathrm{~Gy}(\mathrm{RBE})$ & 100 & $\begin{array}{l}\mathrm{D}_{2 \%}<107 \% \\
\mathrm{D}_{98 \%}>93 \% \\
\mathrm{~V}_{60} \mathrm{~Gy}(\mathrm{RBE}) 100 \%\end{array}$ \\
\hline Rectum & $\begin{array}{l}\text { Max DVH, } 30 \text { Gy(RBE), 30\% } \\
\text { Max DVH, } 50 \text { Gy(RBE), } 20 \% \\
\text { Max DVH, } 60 \text { Gy(RBE), } 10 \%\end{array}$ & $\begin{array}{l}5 \\
5 \\
5\end{array}$ & $\begin{aligned} \mathrm{V}_{30} \mathrm{~Gy}(\mathrm{RBE}) & <30 \% \\
\mathrm{~V}_{50} \mathrm{~Gy}(\mathrm{RBE}) & <20 \% \\
\mathrm{~V}_{60} \mathrm{~Gy}(\mathrm{RBE}) & <10 \%\end{aligned}$ \\
\hline Bladder & $\begin{array}{l}\text { Max DVH, } 50 \text { Gy(RBE), 30\% } \\
\text { Max DVH, } 60 \text { Gy(RBE), 30\% }\end{array}$ & $\begin{array}{l}5 \\
5\end{array}$ & $\begin{array}{l}\mathrm{V}_{50} \mathrm{~Gy}(\mathrm{RBE})<30 \% \\
\mathrm{~V}_{60} \mathrm{~Gy}(\mathrm{RBE})<15 \%\end{array}$ \\
\hline Femoral head & Max dose, 45 Gy(RBE) & 5 & $\mathrm{D}_{\max }<45 \mathrm{~Gy}(\mathrm{RBE})$ \\
\hline Colon and small intestine & Max DVH, 50 Gy(RBE), $0.5 \mathrm{~cm}^{3}$ & 5 & $\mathrm{~V}_{50} \mathrm{~Gy}(\mathrm{RBE})<0.5 \mathrm{~cm}^{3}$ \\
\hline
\end{tabular}

CTV, clinical target volume; DVH, dose-volume histogram; RBE, relative biological effectiveness; $\mathrm{D}_{\max }$, maximum dose.

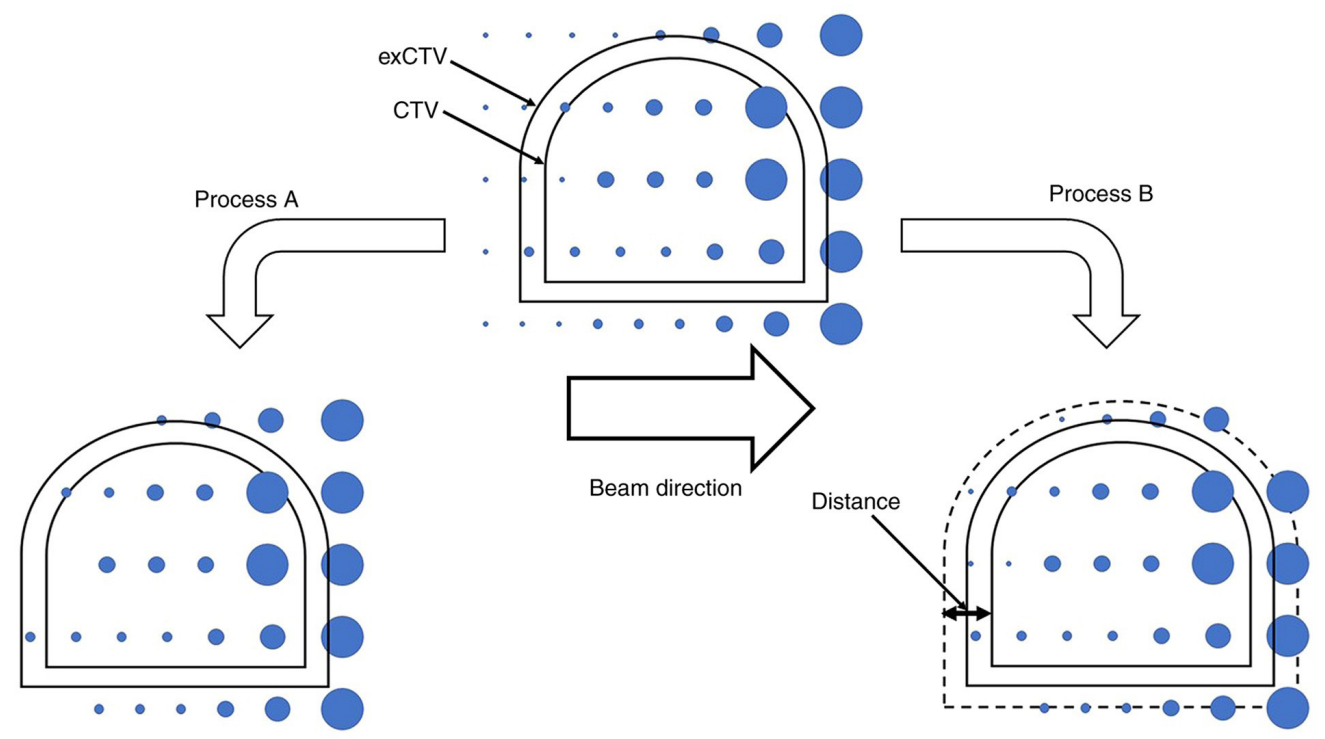

Figure 1. Schematic diagram of process. Process A, the size of the circle represents the relative energy deposit of each spot. The energy was varied from 0.02 to $0.1 \%$. Spots with lower than specified energy deposit were deleted. Process B, the dotted line represents a certain distance from the CTV. Distance was varied from 9 to $13 \mathrm{~mm}$. Spots outside the dotted line were deleted. CTV, clinical target volume; exCTV, expanded clinical target volume.

reduce the $\mathrm{D}_{\max }$ of the rectum, bladder, and urethral bulb in contact with the prostate gland, but high dose volume could be reduced by process $B$. Our results indicated that modification of the initial dose plan by process $\mathrm{B}$, which consisted of deletion of spots at a distance from the CTV can efficiently diminish the high-dose volume of the rectum and bladder, which would be expected to contribute to a reduced likelihood of the occurrence of late toxicities.

To maintain dose homogeneity in the target, and reduction of dose variations inside the target is necessary and hot spots outside the target can be an obstacle $(21,22)$. The spots will inevitably occur outside the CTV for the reason the irradiation dose around each spot is determined by the Gaussian function and there is a distance between the spots and CTV has robustness. As stated in Results, the INH showed substantial increase with the use of process B. The change was particularly prominent when spots that were $13-11 \mathrm{~mm}$ away from the CTV were deleted. These results imply that extra spots at about
$1 \mathrm{~cm}$ or more for the CTV are necessary to maintain the dose homogeneity in the target. On the other hand, use of process A, which deletes spots with lower weighting depositions had not as remarkable change on the INH as process $\mathrm{B}$, thereby the risk of compromising the dose homogeneity of the targe seems rather low.

Synchrotron-based pencil beam scanning delivery systems are very complex and the beam delivery times are affected by multiple variables. The beam delivery times include the layer switch, spot switch, and spot spill times (28). As shown in Fig. 5 the number of layers was smaller in process $\mathrm{B}$ than in process $\mathrm{A}$. On the contrary, the number of spots was smaller in process $\mathrm{A}$ than in process $\mathrm{B}$. Considering that the beam delivery time was much shorter in process $\mathrm{B}$, it appears that the beam delivery time is affected to a greater degree by the number of deleted layers rather than by the number of deleted spots. In fact, 2 patients in whom the beam delivery time was shortened by more than $50 \mathrm{sec}$ showed 
Table III. Summary of data.

\begin{tabular}{|c|c|c|c|c|c|}
\hline \multirow[b]{2}{*}{ Parameters } & \multirow[b]{2}{*}{ Initial plan } & \multicolumn{2}{|c|}{ Process A } & \multicolumn{2}{|c|}{ Process B } \\
\hline & & $\begin{array}{c}\text { All } \\
\text { (energy) }\end{array}$ & $\begin{array}{c}\text { Final } \\
\text { (energy) }\end{array}$ & $\begin{array}{c}\text { All } \\
\text { (distance) }\end{array}$ & $\begin{array}{c}\text { Final } \\
\text { (distance) }\end{array}$ \\
\hline Rectum $V_{50 \text { Gy(RBE) }}$ & $3.5-16.0(11.1 \pm 3.1)$ & $\begin{array}{c}3.1-16.0 \\
(11.5 \pm 3.0)\end{array}$ & $\begin{array}{c}3.1-15.8 \\
(11.3 \pm 3.2)\end{array}$ & $\begin{array}{c}3.4-16.4 \\
(10.4 \pm 2.9)\end{array}$ & $\begin{array}{l}3.4-14.9 \\
(8.9 \pm 2.7)\end{array}$ \\
\hline Rectum $D_{\max } G y(R B E)$ & $\begin{array}{l}54.2-64.0 \\
(63.0 \pm 1.8)\end{array}$ & $\begin{array}{l}53.7-65.2 \\
(63.2 \pm 1.6)\end{array}$ & $\begin{array}{l}53.7-65.2 \\
(63.2 \pm 2.0)\end{array}$ & $\begin{array}{l}54.2-64.3 \\
(63.0 \pm 1.5)\end{array}$ & $\begin{array}{l}54.2-64.2 \\
(62.7 \pm 1.9)\end{array}$ \\
\hline Bladder $\mathrm{V}_{50 \text { Gy(RBE) }}$ & $\begin{array}{c}4.4-17.5 \\
(9.7 \pm 3.6)\end{array}$ & $\begin{array}{c}4.4-18.0 \\
(9.7 \pm 3.6)\end{array}$ & $\begin{array}{c}4.4-17.1 \\
(10.1 \pm 3.7)\end{array}$ & $\begin{array}{c}3.5-17.4 \\
(8.6 \pm 3.2)\end{array}$ & $\begin{array}{c}3.5-16.0 \\
(7.4 \pm 3.0)\end{array}$ \\
\hline Bladder $D_{\max } G y(R B E)$ & $\begin{array}{c}62.6-64.4 \\
(63.6 \pm 0.4)\end{array}$ & $\begin{array}{l}62.4-65.9 \\
(63.6 \pm 0.5)\end{array}$ & $\begin{array}{c}62.4-65.9 \\
(63.8 \pm 0.6)\end{array}$ & $\begin{array}{c}61.2-64.8 \\
(63.6 \pm 0.6)\end{array}$ & $\begin{array}{c}61.2-64.8 \\
(63.5 \pm 0.9)\end{array}$ \\
\hline Urethral bulb $\mathrm{V}_{60} \mathrm{Gy(RBE)}$ & $\begin{array}{l}80.6-100.0 \\
(99.2 \pm 3.5)\end{array}$ & $\begin{array}{l}77.7-100.0 \\
(99.3 \pm 3.4)\end{array}$ & $\begin{array}{l}81.4-100.0 \\
(99.2 \pm 3.4)\end{array}$ & $\begin{array}{l}33.6-100.0 \\
(94.5 \pm 12.7)\end{array}$ & $\begin{array}{l}33.4-100.0 \\
(83.4 \pm 19.7)\end{array}$ \\
\hline 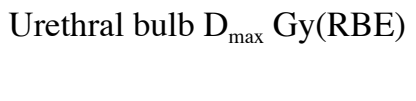 & $\begin{array}{l}63.8-65.5 \\
(64.4 \pm 0.4)\end{array}$ & $\begin{array}{l}63.6-66.0 \\
(64.5 \pm 0.5)\end{array}$ & $\begin{array}{c}63.6-64.7 \\
(64.2 \pm 0.3)\end{array}$ & $\begin{array}{l}62.7-68.7 \\
(64.5 \pm 0.7)\end{array}$ & $\begin{array}{l}62.7-65.0 \\
(63.9 \pm 0.5)\end{array}$ \\
\hline Inhomogeneity index & $\begin{array}{c}0.03-0.10 \\
(0.04 \pm 0.01)\end{array}$ & $\begin{array}{c}0.03-0.10 \\
(0.04 \pm 0.01)\end{array}$ & $\begin{array}{c}0.03-0.10 \\
(0.05 \pm 0.01)\end{array}$ & $\begin{array}{c}0.03-0.14 \\
(0.06 \pm 0.02)\end{array}$ & $\begin{array}{c}0.04-0.14 \\
(0.08 \pm 0.03)\end{array}$ \\
\hline Beam delivery time, sec & $\begin{array}{l}238.4-424.4 \\
(302.6 \pm 47.2)\end{array}$ & $\begin{array}{l}232.8-425.0 \\
(290.2 \pm 38.7)\end{array}$ & $\begin{array}{l}232.8-415.7 \\
(296.9 \pm 46.5)\end{array}$ & $\begin{array}{l}217.2-421.8 \\
(290.4 \pm 45.0)\end{array}$ & $\begin{array}{l}217.2-412.5 \\
(277.6 \pm 44.9)\end{array}$ \\
\hline
\end{tabular}

All data are presented as the range (mean $\pm S D)$. RBE, relative biological effectiveness; $D_{\max }$, maximum dose.

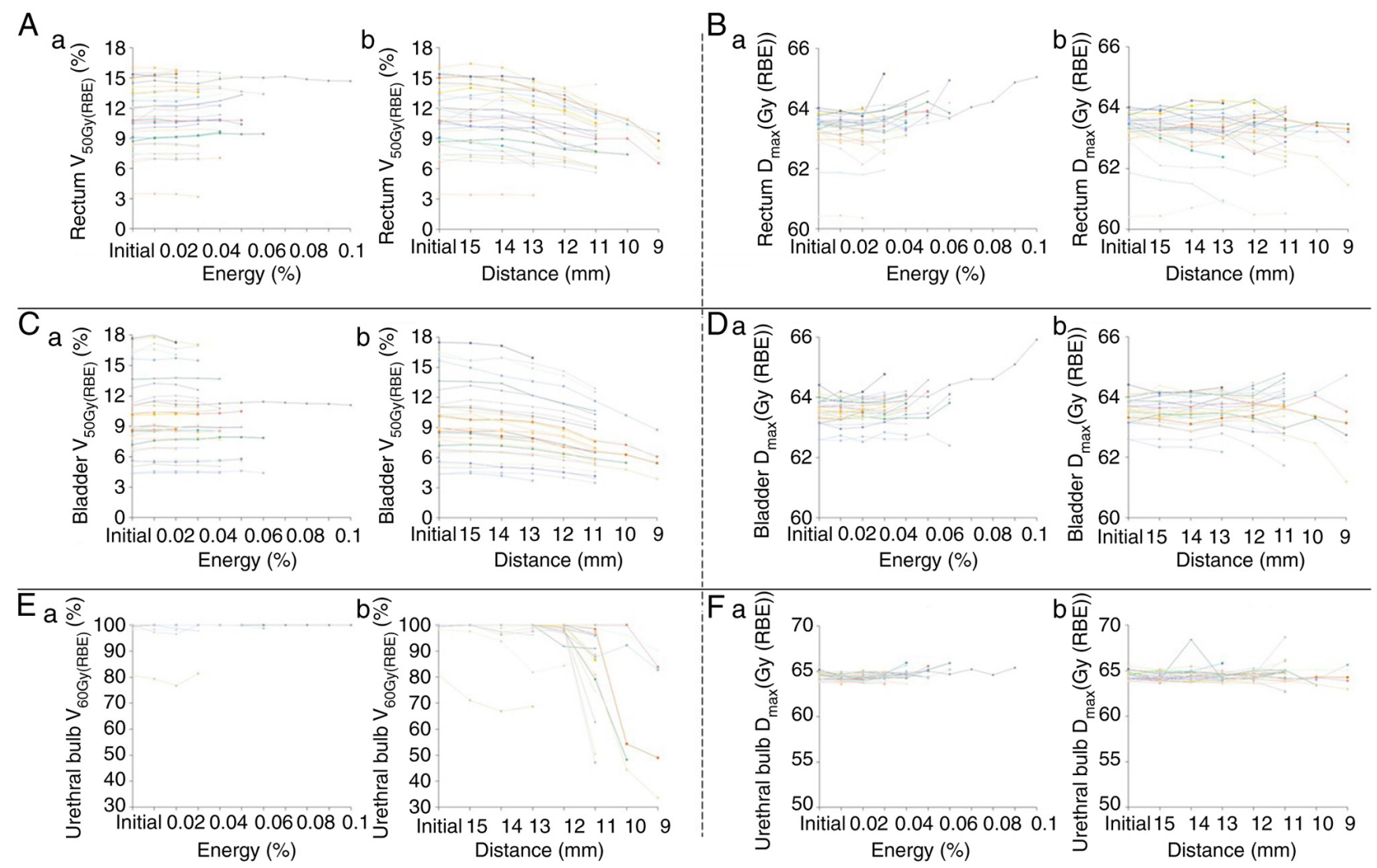

Figure 2. Changes of the doses to the organs at risk. Rectum $V_{50 \text { Gy(RBE) }}$ of (A-a) process $A$ and (A-b) process $B$. Rectum $D_{\max }$ of (B-a) process $A$ and (B-b) process B. Bladder $\left.\mathrm{V}_{50 \mathrm{~Gy}} \mathrm{RBE}\right)$ of (C-a) process $\mathrm{A}$ and (C-b) process B. Bladder $\mathrm{D}_{\max }$ of (D-a) process $\mathrm{A}$ and (D-b) process $\mathrm{B}$. Urethral bulb $\mathrm{V}_{60 \mathrm{~Gy}}(\mathrm{RBE})$ of (E-a) process A and (E-b) process B. Urethral bulb $\mathrm{D}_{\max }$ of (F-a) process A and (F-b) process B. RBE, relative biological effectiveness; $\mathrm{D}_{\max }$, maximum dose. 

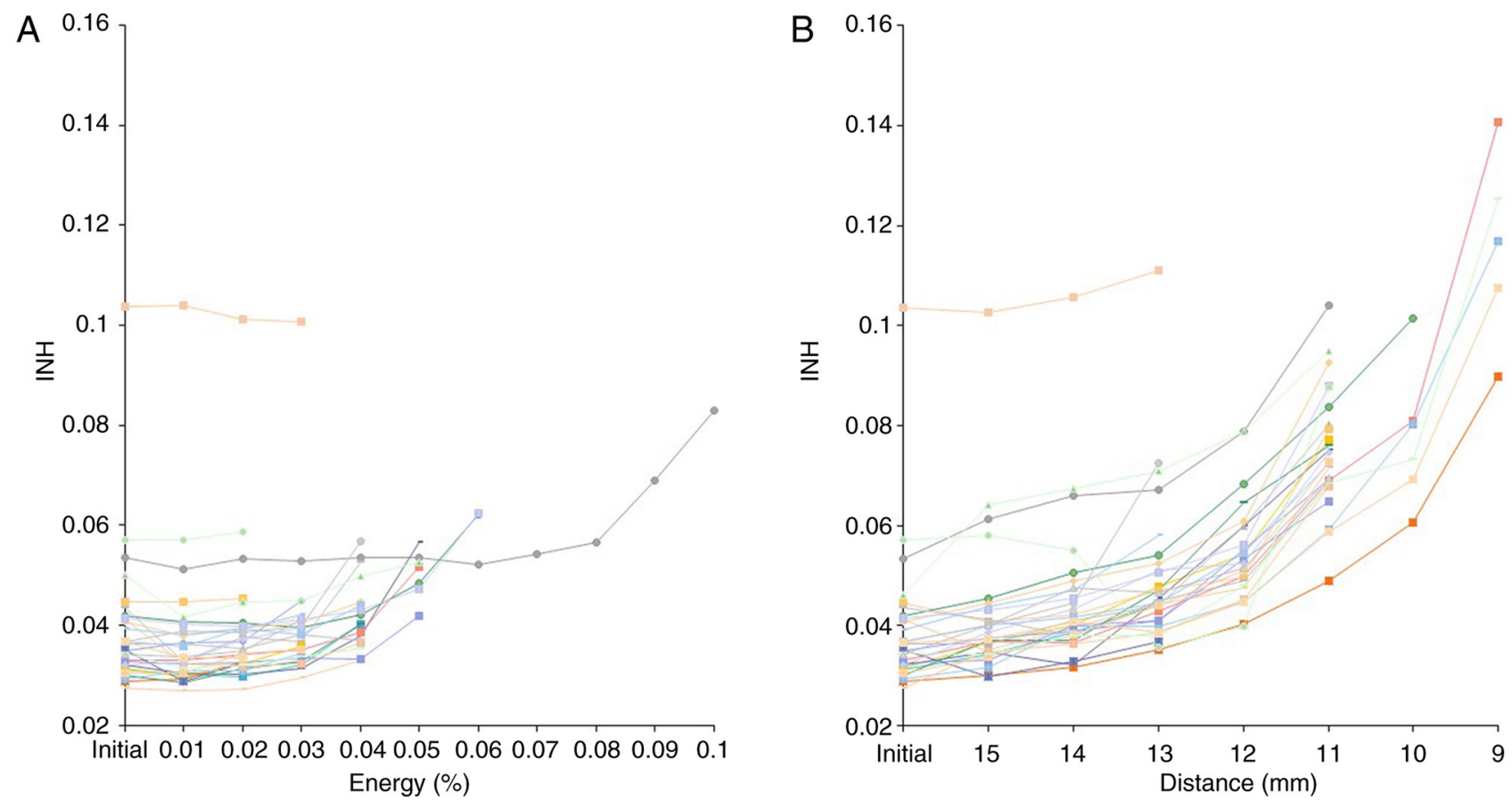

Figure 3. Changes of the INH. The INH of A: process A and B: process B. INH, inhomogeneity index.
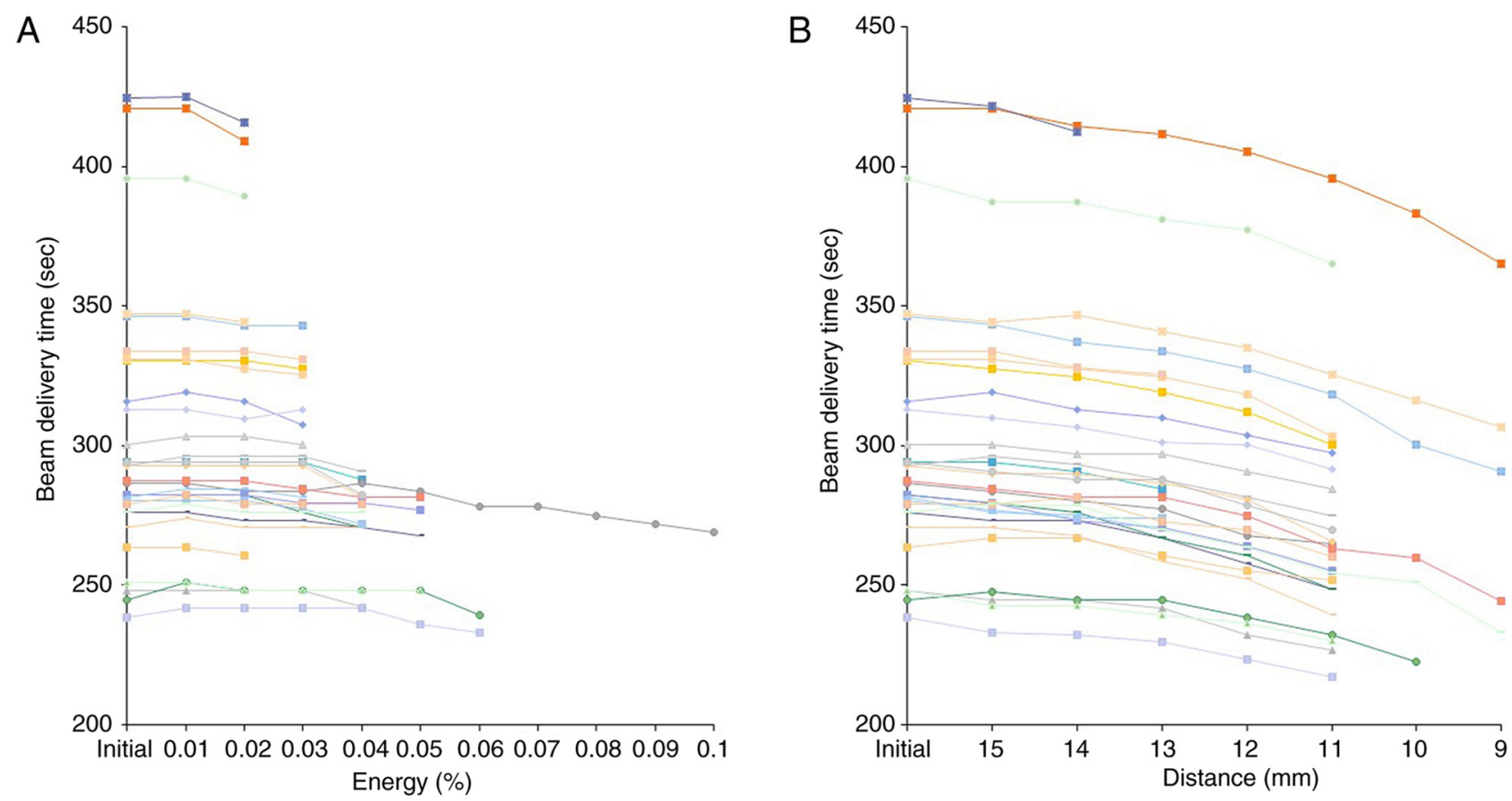

Figure 4. Changes of beam delivery time. The beam delivery time of A: process A and B: process B.

deletion of 2 and 4 layers, respectively. The most important significance of beam delivery time shortening is the possibility of stabilized daily practice of treatment. Usually, patients are obliged to lay still during the position set-up and beam delivery with patience while the urinary bladder is filling up. As the treatment time progresses, it becomes difficult to hold the desire for urination. Although not too often, it becomes necessary in some cases to stop the radiation to allow the patients to go to the bathroom to avoid leakage of urine in the treatment room. Shortening trend of the beam delivery time, as by the use of process B, can help in stabilizing the daily practice of treatment. Another important benefit of beam delivery time shortening is improvement of the throughput of facilities. In Japan, according to a 2018 survey, approximately 1700 prostate cancer patients are treated at 19 particle beam facilities (89.5 patients per facility on average; not disclosed in the data collected by the particle beam medical facilities), which is equivalent to 1879 treatment times by the 21 -fraction 

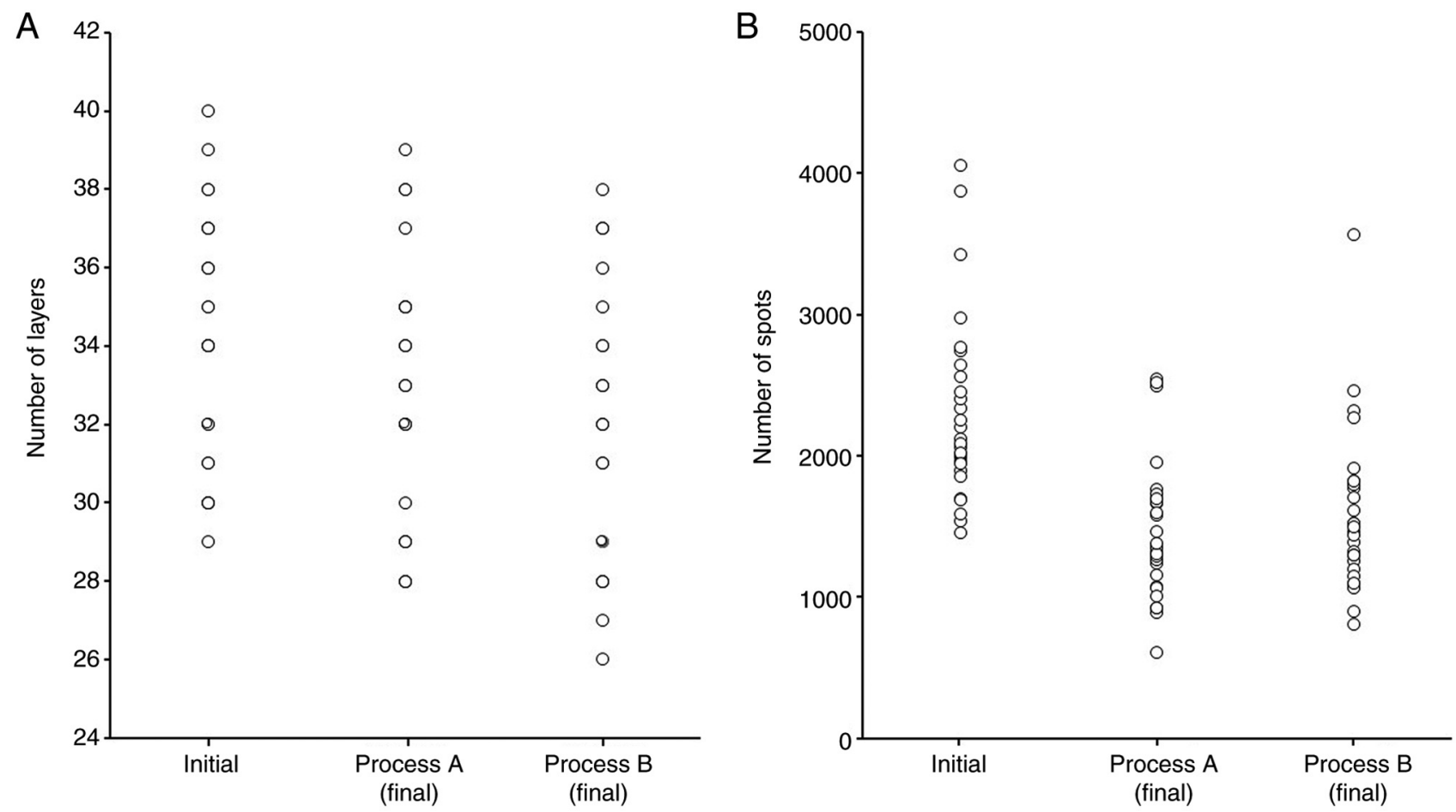

Figure 5. Numbers of layers and spots. (A) Number of layers. (B) Number of spots.

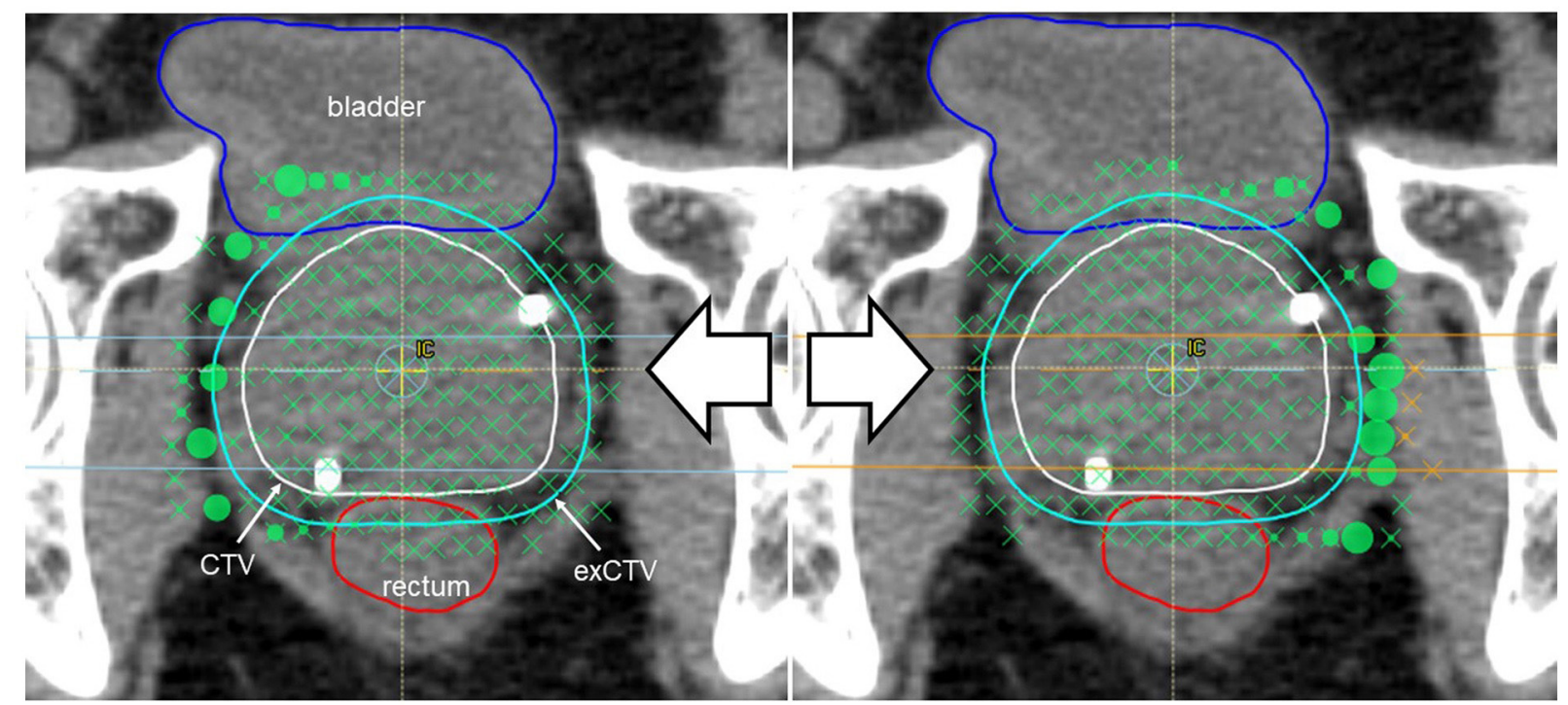

Figure 6. Energy deposit of each beam. Cross marks and circle sizes represent the location and energy deposit of the spots. Arrowheads show the beam directions. CTV, clinical target volume; exCTV, expanded clinical target volume.

protocol and 3400 times by the 38 -fraction protocol per facility per year. The possible extent of beam delivery time shortening by the use of process $\mathrm{A}$ is $3 \mathrm{~h}$ in the 21-fraction protocol and $5.4 \mathrm{~h}$ in the 38 -fraction protocol, and that by the use of process $\mathrm{B}$ is $13 \mathrm{~h}$ in the 21 -fraction protocol and $23.6 \mathrm{~h}$ in the 38-fraction protocol.

Before we started this study, we thought that the optimization operation would result in high-weighted deposition spots becoming densely gathered inside the CTV and low-weighted deposition spots becoming scattered sparsely outside the CTV. However, actually, relatively high-weighted deposition spots for each beam were located in the area beyond the CTV and relatively low-weighted deposition spots were scattered in and around the CTV as shown in Fig. 6. As stated in Results, modification of the plan by process $B$ was effective for reducing the dose to the OAR, whereas that by process A had little effect on the dose to the OAR, implying that relatively low-weighted deposition spots are abundantly scattered not only outside, but also inside the CTV after the optimization operation. The merits of using process $\mathrm{B}$ are reduction of the dose volumes to the OAR, while an important demerit is the loss of dose homogeneity in the target. An optimal cutoff range should be determined based 

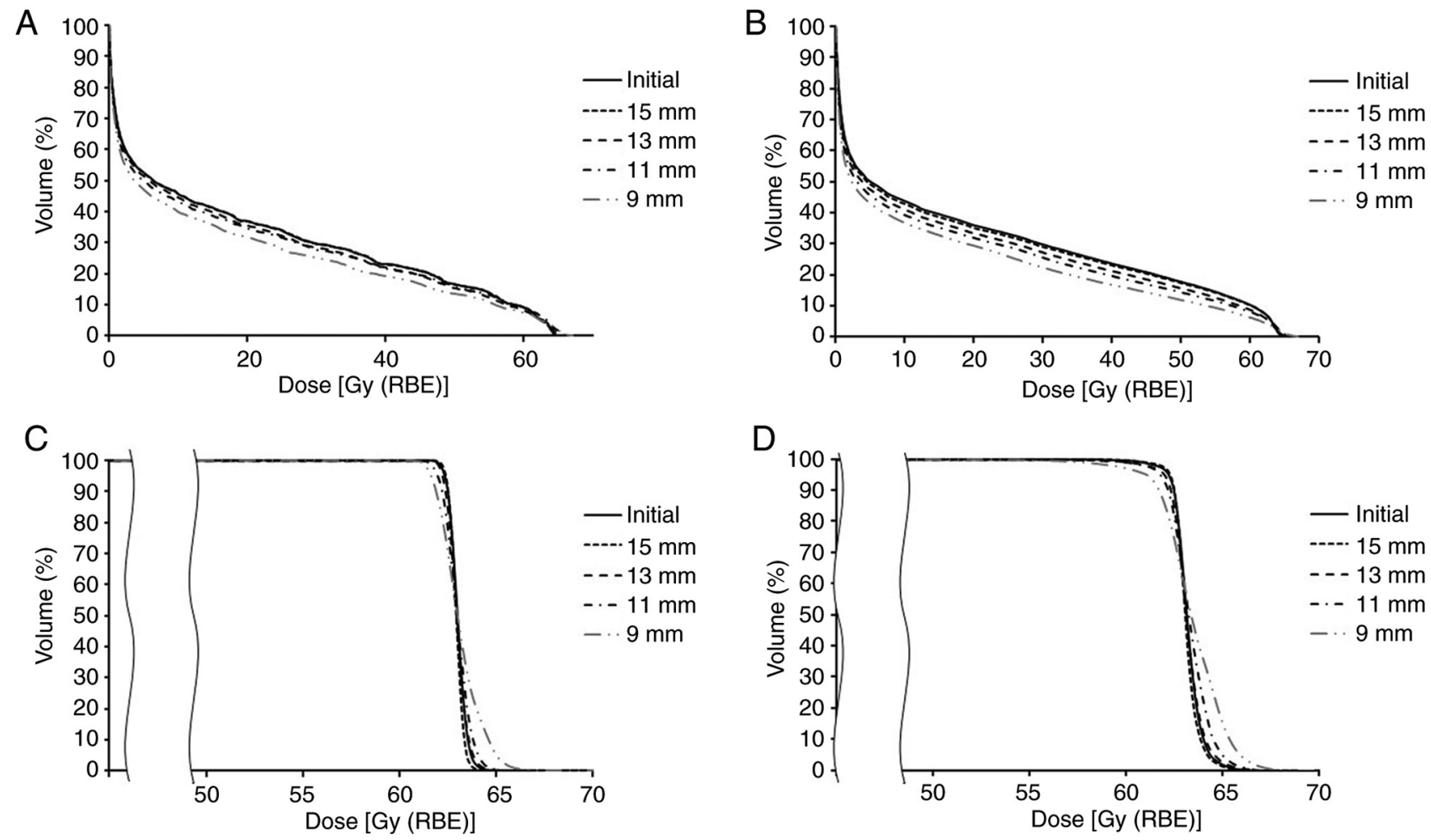

Figure 7. Dose-volume histogram. Dose-volume of the (A) rectum, (B) bladder, (C) CTV and (D) exCTV. CTV, clinical target volume; exCTV, expanded clinical target volume; RBE, relative biological effectiveness.
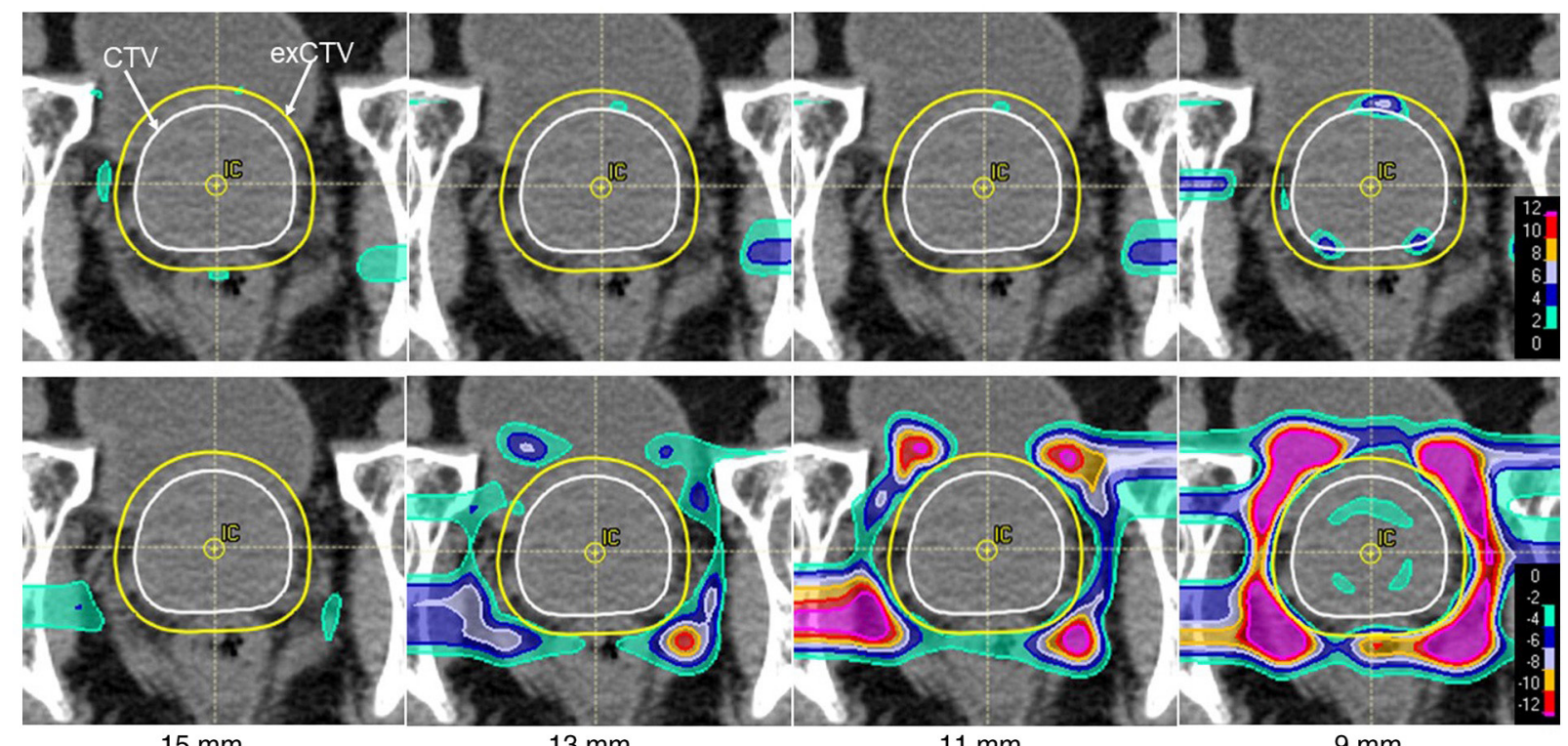

Figure 8. Subtraction dose distribution image to the initial plan. Upper panel, dose elevated area. Range represents the percentile of the prescription dose. Lower panel, dose reduced area. CTV, clinical target volume; exCTV, expanded clinical target volume.

on the priorities set by the attending physician. On the other hand, use of process A seemed to have little clinical effect.

No study has attempted to improve the treatment planning using the same methods as ours, but some studies have focused on similar ideas. First, the challenge of the plan quality using direct spot reduction. Researchers in Paul Scherrer Institute, Switzerland reported spot reduction with their in-house TPS which allowed randomly selected pencil beams with lower weights to be excluded and revealed that the plan quality was maintained or even improved using this technique $(11,12)$. They also compared it with the commercial TPS (Eclipse ${ }^{\mathrm{TM}}$ ) and reported that the commercial TPS could cover the same target volume by reducing the spots to $1 / 3$ or less (29). Second, application of a collimator and aperture system. The lateral size of a proton pencil beam, or spot, is characterized by the 
Gaussian $\sigma$ of the lateral distribution. Past studies have shown that the quality of spot scanning PBT strongly depends on the spot $\sigma$ and spot placements $(13,14)$. The lateral penumbra of an individual field of spot scanning beams is not usually sharper than that of passively scattered beams $(30,31)$. The lateral penumbra can be reduced using a collimator and aperture (13,15-17). Moreover, Hyer et al developed a dynamic collimation system which shaped the lateral extent of the beam separately for each energy layer (6). This system separates the target into individual layers and sets the collimator individually, thus making it possible to reduce the penumbra of each layer, even for complex-shaped targets. Third, combining beams of various energies. Multiple energy extraction (MEE) is an advanced technology which was originally developed at Heavy Ion Medical Accelerator, Chiba, and has been incorporated in the Hitachi's PBT system (32). Younkin et al reported that MEE could shorten the beam delivery time by an average of $35 \%$ as compared to conventional method (18). The advantage of our method is simple. The parameters for optimization can be set in a large variety of combinations, but that also makes it difficult to decide whether to continue with the plan optimization or accept the current solution as the final treatment plan. The spot deletion technique can be implemented in a few steps to easily and directly approach the ideal dose distribution. Moreover, it can help in achieving both OAR dose reduction and shortening of the beam delivery time. In addition, it is extremely versatile in that a commercial TPS can be used and that no special equipment or expensive capital investment is needed.

Other than spot editing technique, spacer implantation is known to reduce the dose of the rectum. The SpaceOAR $^{\circledR}$ System (Augmenix, Inc.) is the only Food and Drug Administration-approved absorbable polyethylene glycol (PEG) hydrogel available in the market that can be introduced between the prostate gland and the rectum to decrease the toxicity and minimize the changes in the quality of life (QOL) occurring after radiotherapy for prostate cancer (33-35). In Japan, it was approved for coverage by the National Health Insurance in 2018. Space OAR implantation was already installed in our hospital. We examined whether the process $B$ could reduce the dose of the rectum in 8 patients with space OAR implantation as adding trial. As shown in the results, process $\mathrm{B}$ was able to reduce $\mathrm{V}_{50 \mathrm{~Gy}(\mathrm{RBE})}$ and $\mathrm{D}_{\max }$ of the rectum, and $\mathrm{D}_{95}$ of the CTV was almost maintaind to the level of $100 \%$. We consider this spots deleting technique can reduce the irradiation dose of the rectum while maintaining the CTV dose even patients with spce OAR is implanted.

Using this spots deletion technique, avoiding the adverse events and maintaining of the patients' QOL is ideal. Late adverse events which was radiation induced proctitis (grade 2 in Common Terminology Criteria for Adverse Events, ver 4.0) were found in 1 patient among the 12 patients who were treated before this study using conventional method. While, 1 patients among the 30 patients of this study suffered from grade 2 radiation induced procitis. Kobe Proton Center is a new facility and does not have abundant data treated with conventional method. We plan to examine the clinical usefulness of spots deletion technique with more number of patients and longer follow-up periods, comparing with some historical literature data.

Operation time is an issue that needs to be considered and largely depends on the spot deletion procedure. Process A can be implemented within a few seconds because RayStation can delete spots with a weight greater than any threshold value all at once with just one operation. On the other hand, process $B$ needs manual operation on the beams-eye-view image that takes about 10-20 min and much labor. Automatic spot deletions using parameter settings could make the process smooth and quick. Spot editing still has room for improvement. We used only the spot deleting technique in this study. Process B allowed dose reduction to the OAR, but at the expense of the dose homogeneity at the target. RayStation has the capability of editing each spot not only by deleting, but also by adding, moving and multiplying the energy levels. It is expected that with full use made of the many available functions, more ideal planning can be accomplished.

We propose this spot deletion technique in that it can directly reflect the physician's intentions in the treatment plan, such as reducing the OAR dose while maintaining the CTV dose with a simple operation even after optimization procedure.

In conclusion, modification of the treatment plan by deleting the spots that are distant from the target can result in dose reduction to the OAR in spot scanning PBT for prostate cancer.

\section{Acknowledgements}

Not applicable.

\section{Funding}

This study was supported by the Grant-in-Aid for Challenging Research of Exploratory (grant no. 21K19449).

\section{Availability of data and materials}

The datasets used and/or analyzed during the current study are available from the corresponding author on reasonable request.

\section{Authors' contributions}

NF, TH and TY conceived and designed the study. MM, YD and TSu performed the simulation study for dose calculation. NF and TSo analyzed the data and wrote the manuscript. NF and TSo confirm the authenticity of all the raw data. All authors read and approved the final manuscript.

\section{Ethics approval and consent to participate}

All study procedures involving human participants were conducted in accordance with the ethical standards of the Kobe Proton Center research committee (Kobe, Japan) and in compliance with the Declaration of Helsinki, and were approved by the Kobe Proton Center institutional review board (approval no. 30-07). Informed consent was waived as this was a retrospective simulation study. 


\section{Patient consent for publication}

Not applicable.

\section{Competing interests}

The authors declare that they have no competing interests.

\section{References}

1. Lawrence JH, Tobias CA, Born JL, Linfoot JA, Kling RP and Gottschalk A: Alpha and proton heavy particles and the bragg peak in therapy. Trans Am Clin Climatol Assoc 75: 111-116, 1964.

2. Bortfeld T and Schlegel W: An analytical approximation of depth-dose distributions for therapeutic proton beams. Phys Med Biol 41: 1331-1339, 1996.

3. Schulte RW, Slater JD, Rossi CJ Jr and Slater JM: Value and perspectives of proton radiation therapy for limited stage prostate cancer. Strahlenther Onkol 176: 3-8, 2000.

4. Nihei K, Ogino T, Onozawa M, Onozawa M, Murayama S, Fuji H, Murakami M and Hishikawa Y: Multi-institutional phase II study of proton beam therapy for organ-confined prostate cancer focusing on the incidence of late rectal toxicities. Int J Radiat Oncol Biol Phys 81: 390-396, 2011.

5. Royce TJ and Efstathiou JA: Proton therapy for prostate cancer: A review of the rationale, evidence, and current state. Urol Oncol 37: 628-636, 2019.

6. Hyer DE, Hill PM, Wang D, Smith BR and Flynn RT: A dynamic collimation system for penumbra reduction in spot-scanning proton therapy: Proof of concept. Med Phys 41: 091701, 2014

7. DeLaney TF: Proton therapy in the clinic. Front Radiat Ther Oncol 43: 465-485, 2011

8. Bert C and Durante M: Motion in radiotherapy: Particle therapy. Phys Med Biol 56: R113-R144, 2011.

9. Pugh TJ, Munsell MF, Choi S, Nguyen QN, Mathai B, Zhu XR, Sahoo N, Gillin M, Johnson JL, Amos RA, et al: Quality of life and toxicity from passively scattered and spot-scanning proton beam therapy for localized prostate cancer. Int J Radiat Oncol Biol Phys 87: 946-953, 2013.

10. Widesott L, Pierelli A, Fiorino C, Lomax AJ, Amichetti M, Cozzarini C, Soukup M, Schneider R, Hug E, Di Muzio N, et al: Helical tomotherapy vs. intensity-modulated proton therapy for whole pelvis irradiation in high-risk prostate cancer patients: Dosimetric, normal tissue complication probability, and generalized equivalent uniform dose analysis. Int J Radiat Oncol Biol Phys 80: 1589-1600, 2011.

11. van de Water S, Kraan AC, Breedveld S, Schillemans W, Teguh DN, Kooy HM, Madden TM, Heijmen BJ and Hoogeman MS: Improved efficiency of multi-criteria IMPT treatment planning using iterative resampling of randomly placed pencil beams. Phys Med Biol 58: 6969-6983, 2013.

12. van de Water S, Safai S, Schippers JM, Weber DC and Lomax AJ: Towards FLASH proton therapy: The impact of treatment planning and machine characteristics on achievable dose rates. Acta Oncol 58: 1463-1469, 2019.

13. Wang D, Smith BR, Gelover E, Flynn RT and Hyer DE: A method to select aperture margin in collimated spot scanning proton therapy. Phys Med Biol 60: N109-N119, 2015.

14. Meier G, Leiser D, Besson R, Mayor A, Safai S, Weber DC and Lomax AJ: Contour scanning for penumbra improvement in pencil beam scanned proton therapy. Phys Med Biol 62: 2398-2416, 2017.

15. Winterhalter C, Meier G, Oxley D, Weber DC, Lomax AJ and Safai S: Contour scanning, multi-leaf collimation and the combination thereof for proton pencil beam scanning. Phys Med Biol 64: 015002, 2018.

16. Bues M, Newhauser WD, Titt U and Smith AR: Therapeutic step and shoot proton beam spot-scanning with a multi-leaf collimator: A Monte Carlo study. Radiat Prot Dosimetry 115: 164-169, 2005.

17. Dowdell SJ, Clasie B, Depauw N, Metcalfe P, Rosenfeld AB, Kooy HM, Flanz JB and Paganetti H: Monte Carlo study of the potential reduction in out-of-field dose using a patient-specific aperture in pencil beam scanning proton therapy. Phys Med Biol 57: 2829-2842, 2012.

18. Younkin JE, Bues M, Sio TT, Liu W, Ding X, Keole SR, Stoker JB and Shen J: Multiple energy extraction reduces beam delivery time for a synchrotron-based proton spot-scanning system. Adv Radiat Oncol 3: 412-420, 2018.
19. Zhang X, Penagaricano J, Narayanasamy G, Corry P, Liu T, Sanjay M, Paudel N and Morrill S: Helical tomotherapy to LINAC plan conversion utilizing RayStation Fallback planning. J Appl Clin Med Phys 18: 178-185, 2017.

20. International Commission on Radiation Units \& Measurements (ICRU): Prescribing, recording, and reporting proton-beam therapy. ICRU 7: Report 78. ICRU, Bethesda, MD, 2007.

21. Zhu XR, Poenisch F, Li H, Zhang X, Sahoo N, Wu RY, Li X, Lee AK, Chang EL, Choi S, et al: A single-field integrated boost treatment planning technique for spot scanning proton therapy. Radiat Oncol 9: 202, 2014.

22. Kirk ML, Tang S, Zhai H, Vapiwala N, Deville C, James P, Bekelman JE, Christodouleas JP, Tochner Z and Both S: Comparison of prostate proton treatment planning technique, interfraction robustness, and analysis of single-field treatment feasibility. Pract Radiat Oncol 5: 99-105, 2015.

23. Hou Z, Li G and Bai S: High dose versus conventional dose in external beam radiotherapy of prostate cancer: A meta-analysis of long-term follow-up. J Cancer Res Clin Oncol 141: 1063-1071, 2015.

24. Jolnerovski M, Salleron J, Beckendorf V, Peiffert D, Baumann AS, Bernier V, Huger S, Marchesi V and Chira C: Intensity-modulated radiation therapy from 70 to $80 \mathrm{~Gy}$ in prostate cancer: Six-year outcomes and predictors of late toxicity. Radiat Oncol 12: 99, 2017.

25. Thor M, Deasy JO, Paulus R, Robert Lee W, Amin MB Bruner DW, Low DA, Shah AB, Malone SC, Michalski JM, et al: Tolerance doses for late adverse events after hypofractionated radiotherapy for prostate cancer on trial NRG oncology/RTOG 0415. Radiother Oncol 135: 19-24, 2019.

26. Colaco RJ, Hoppe BS, Flampouri S, McKibben BT, Henderson RH, Bryant C, Nichols RC, Mendenhall WM, Li Z, Su Z, et al: Rectal toxicity after proton therapy for prostate cancer: An analysis of outcomes of prospective studies conducted at the university of Florida proton therapy institute. Int J Radiat Oncol Biol Phys 91: 172-181, 2015.

27. Boladeras A, Ferrer F, Navarro V, De Blas R, Cunillera O, Mateo D, Gutierrez C, Martinez E, Villà S, Pera J, et al: Association between EBRT dose volume histograms and quality of life in prostate cancer patients. Rep Pract Oncol Radiother 23: 360-368, 2018

28. Shen J, Tryggestad E, Younkin JE, Keole SR, Furutani KM, Kang Y, Herman MG and Bues M: Technical note: Using experimentally determined proton spot scanning timing parameters to accurately model beam delivery time. Med Phys 44: 5081-5088, 2017.

29. Rosas S, Belosi FM, Bizzocchi N, Böhlen T, Zepter S, Morach P, Lomax AJ, Weber DC and Hrbacek J: Benchmarking a commercial proton therapy solution: The paul scherrer institut experience. Br J Radiol 93: 20190920, 2020.

30. Schippers JM and Lomax AJ: Emerging technologies in proton therapy. Acta Oncol 50: 838-850, 2011.

31. Engelsman M, Schwarz M and Dong L: Physics controversies in proton therapy. Semin Radiat Oncol 23: 88-96, 2013.

32. Mizushima K, Katagiri K, Iwata Y, Furukawa T, Fujimoto T, Sato S, Hara Y, Shirai T and Noda K: Experimental studies of systematic multiple-energy operation at HIMAC synchrotron. Nucl Instrum Methods Phys Res Sec B 331: 243-247, 2014.

33. Hamstra DA, Mariados N, Sylvester J, Shah D, Karsh L, Hudes R, Beyer D, Kurtzman S, Bogart J, His RA, et al: Continued benefit to rectal separation for prostate radiation therapy: Final results of a phase III trial. Int J Radiat Oncol Biol Phys 97: 976-985, 2017.

34. Karsh LI, Gross ET, Pieczonka CM, Aliotta PJ, Skomra CJ, Ponsky LE, Nieh PT, Han M, Hamstra DA and Shore ND: Absorbable hydrogel spacer use in prostate radiotherapy: A comprehensive review of phase 3 clinical trial published data. Urology 115: 39-44, 2018.

35. Wei JT, Dunn RL, Litwin MS, Sandler HM and Sanda MG: Development and validation of the expanded prostate cancer index composite (EPIC) for comprehensive assessment of health-related quality of life in men with prostate cancer. Urology 56: 899-905, 2000.

This work is licensed under a Creative Commons Attribution-NonCommercial-NoDerivatives 4.0 International (CC BY-NC-ND 4.0) License. 